\title{
Estimation of Turbulent Dissipation Rate Using 2D Data in Channel Flows
}

\author{
Dinesh Bhatia $^{1}$, Callum Atkinson ${ }^{1}$, Vassili Kitsios ${ }^{1}$, Nicolas Mazellier ${ }^{3}$, Julio Soria ${ }^{1,2}$ \\ ${ }^{1}$ Department of Mechanical and Aerospace Engineering, Laboratory for Turbulence Research in Aerospace and \\ Combustion, Monash University \\ Melbourne, Australia \\ dinesh.bhatia@monash.edu; callum.atkinson@monash.edu \\ ${ }^{2}$ Department of Aeronautical Engineering, King Abdulaziz Univeristy \\ Jeddah, Kingdom of Saudi Arabia \\ ${ }^{3}$ Universit'e d'Orleans, INSA-CVL \\ PRISME EA 4229, F45072, Orleans, France
}

\begin{abstract}
The dissipation rate of turbulent kinetic energy is a key parameter in the analysis of turbulent flows and its accurate determination in experiments is of paramount importance to turbulence research. In order to calculate turbulence dissipation rate, values of all the components of fluctuating velocity gradient tensor are required. Experimental methods, in particular 2D planar Particle Image Velocimetry (PIV), cannot measure velocity components in all three spatial dimensions, hence dissipation cannot be calculated completely from the data provided by such methods. This paper uses data from a Direct Numerical Simulation (DNS) to compare the true turbulent dissipation in a channel flow with its estimates calculated from various models available in literature. These models are shown to fail near the wall, where a substantial fraction of the turbulent kinetic energy is dissipated, significantly limiting estimates of total turbulent dissipation in the flow. This study aims to develop an improved model to estimate turbulent dissipation rate where information limited velocity field information is available especially in the near wall region.
\end{abstract}

Keywords: turbulent dissipation rate, PIV, experiments, dissipation estimation models

\section{Introduction}

The dissipation rate of kinetic energy by viscous and turbulent stresses plays a major role in turbulent flows. Mostly dominated by small scale turbulence, it is a key parameter in a number of processes such as chemical mixing, combustion or heat exchange. Hence its modelling is of paramount importance in the purpose of prediction and design of industrial systems. However, the estimation of the turbulent dissipation rate still remains inaccessible to most of current measurement techniques which are often limited by their spatial resolution, while in the case of numerical simulations, whether using Large Eddy Simulations (LES) or RANS based flow solvers, knowledge of various factors affecting turbulent dissipation rate is critical in modelling the effects of the unresolved scales of turbulent fluctuations. Thus it is imperative that the turbulent dissipation rate is accurately determined in turbulence research.

To determine turbulent dissipation rate often Direct Numerical Simulations (DNS) of turbulent flows are used, which resolve the entire range of spatial and temporal scales in the flow. They are able to accurately calculate turbulent dissipation rate but are limited by the computational capacity and it is prohibitively expensive to use them to get solutions at high Reynolds numbers, which are typically encountered in the industry and the atmosphere. Experimental methods which are extensively used in the study of turbulent flows at high Reynolds numbers are of lower fidelity by virtue of the limited number of components of the flow velocity and the scales that can be resolved. Hence the accurate determination of turbulent dissipation rate from such data poses a significant challenge. To address the challenges faced, let us look at the equation for turbulent dissipation (Eq. (1))

$$
\varepsilon=2 v\left\langle s_{i j} s_{i j}\right\rangle
$$


where $<\cdot>$ is the ensemble averaging operation and $s_{i j}$ is the turbulent strain rate tensor associated with the turbulent velocity fluctuations and is given by

$$
s_{i j}=\frac{1}{2}\left(\frac{\partial u_{i}^{\prime}}{\partial x_{j}}+\frac{\partial u_{j}^{\prime}}{\partial x_{i}}\right)
$$

where $u_{\mathrm{i}}^{\prime}$ is the turbulent fluctuating velocity along the $i^{\text {th }}$ direction. Upon substitution of Eq. (2) in Eq. (1) and expansion of the terms, the turbulent dissipation rate is given by Eq.(3)

$$
\begin{aligned}
\varepsilon=v\left\langle 2\left(\frac{\partial u^{\prime}}{\partial x}\right)^{2}+2\left(\frac{\partial v^{\prime}}{\partial y}\right)^{2}+2\left(\frac{\partial w^{\prime}}{\partial z}\right)^{2}+\right. & \left(\frac{\partial u^{\prime}}{\partial y}\right)^{2}+\left(\frac{\partial v^{\prime}}{\partial x}\right)^{2}+\left(\frac{\partial w^{\prime}}{\partial x}\right)^{2}+\left(\frac{\partial u^{\prime}}{\partial z}\right)^{2}+\left(\frac{\partial v^{\prime}}{\partial z}\right)^{2} \\
& \left.+\left(\frac{\partial w^{\prime}}{\partial y}\right)^{2}+2\left(\frac{\partial u^{\prime}}{\partial y} \frac{\partial v^{\prime}}{\partial x}\right)+2\left(\frac{\partial u^{\prime}}{\partial z} \frac{\partial w^{\prime}}{\partial x}\right)+2\left(\frac{\partial v^{\prime}}{\partial z} \frac{\partial w^{\prime}}{\partial y}\right)\right\rangle
\end{aligned}
$$

where $\mathrm{x}, \mathrm{y}$ and $\mathrm{z}$ are the streamwise, wall-normal and spanwise directions respectively and $u^{\prime}, v^{\prime}$ and $w^{\prime}$ are the corresponding turbulent fluctuating velocities along those directions.

To calculate the dissipation rate of a turbulent flow, accurate measurement of all nine components of the fluctuating velocity gradient tensor is required. Particle Image Velocimetry (PIV) is extensively used in experiments to investigate turbulent flows, commonly involving planar (2D) measurements of the flow. In such cases, only 5 out of 9 components of the velocity gradient tensor are directly available in an incompressible flow. Furthermore, low-pass filtering induced by PIV results in underestimation of dissipation but this issue is beyond the scope of this study.

A number of methods are available in the literature to calculate turbulent dissipation rate in the absence of some of the components of the velocity gradient tensor [3-8] and have shown varying degree of accuracy in different flows as well as in different regions of a given flow configuration $[5,7,8]$. The measurement plane in case of wall bounded turbulent flows while using planar PIV technique is generally chosen as the $\mathrm{x}-\mathrm{y}$ plane, where $\mathrm{x}$ and $\mathrm{y}$ are the streamwise and wall-normal directions respectively. Various assumptions lead to different forms for turbulent dissipation rate, the most prevalent of which are discussed below by assuming the measurement plane to be the $\mathrm{x}-\mathrm{y}$ plane.

\subsection{Local Axisymmetry Assumption}

According to this assumption, statistical measures of the turbulent quantities are locally axisymmetric about a given axis [3]. If the axis of rotational symmetry is defined as $\mathrm{x}$, the estimated dissipation rate from local isotropy assumption (eLA) takes the following form (Eq. (4))

$$
\varepsilon_{L A}=v\left\langle-\left(\frac{\partial u^{\prime}}{\partial x}\right)^{2}+8\left(\frac{\partial v^{\prime}}{\partial y}\right)^{2}+2\left(\frac{\partial u^{\prime}}{\partial y}\right)^{2}+2\left(\frac{\partial v^{\prime}}{\partial x}\right)^{2}\right\rangle
$$

Although this model is known to be successful in free shear flows, its success in wall bounded flows needs to be evaluated. In the near wall region, the presence of large mean shear along wall normal direction disallows various velocity components and hence their derivatives from being locally axisymmetric about streamwise $\mathrm{x}$ direction [5]. Accordingly, if valid, this model is expected to hold only in regions far enough from the wall.

\subsection{Assumption of Weak Local Isotropy}

The weak local isotropy formulation [9] assumes that the out-of-plane cross gradients have the same magnitude as averaged in-plane cross gradients. 


$$
\left\langle\left(\frac{\partial u^{\prime}}{\partial z}\right)^{2}\right\rangle=\left\langle\left(\frac{\partial w^{\prime}}{\partial x}\right)^{2}\right\rangle=\left\langle\left(\frac{\partial v^{\prime}}{\partial z}\right)^{2}\right\rangle=\left\langle\left(\frac{\partial w^{\prime}}{\partial y}\right)^{2}\right\rangle=\frac{1}{2}\left\langle\left(\frac{\partial u^{\prime}}{\partial y}\right)^{2}+\left(\frac{\partial v^{\prime}}{\partial x}\right)^{2}\right\rangle
$$

and mixed derivative terms of the dissipation equation (Eq. (3)) are equal to each other

$$
\left\langle\frac{\partial u^{\prime}}{\partial z} \frac{\partial w^{\prime}}{\partial x}\right\rangle=\left\langle\frac{\partial v^{\prime}}{\partial z} \frac{\partial w^{\prime}}{\partial y}\right\rangle=\left\langle\frac{\partial u^{\prime}}{\partial y} \frac{\partial v^{\prime}}{\partial x}\right\rangle
$$

Along with continuity, the formula for estimation of turbulence dissipation rate by weak local isotropy formulation $\left(\varepsilon_{W I}\right)$ becomes

$$
\varepsilon_{W I}=v\left\langle 4\left(\frac{\partial u^{\prime}}{\partial x}\right)^{2}+4\left(\frac{\partial v^{\prime}}{\partial y}\right)^{2}+3\left(\frac{\partial u^{\prime}}{\partial y}\right)^{2}+3\left(\frac{\partial v^{\prime}}{\partial x}\right)^{2}+4\left(\frac{\partial u^{\prime}}{\partial x} \frac{\partial v^{\prime}}{\partial y}\right)+6\left(\frac{\partial u^{\prime}}{\partial y} \frac{\partial v^{\prime}}{\partial x}\right)\right\rangle
$$

Local axisymmetry and weak local isotropy formulations are the most successful models found in literature. These models calculate dissipation directly from fluctuating velocity derivatives, hence called 'direct' models. In this paper, the validity of various 'direct' models has been evaluated in wall bounded turbulent flows by using the data from low Reynold's number DNS of turbulent channel flow. The regions of incorrect estimate of turbulent dissipation rate as calculated by these models are highlighted and a new model is proposed with improved performance.

\section{Results and Discussion}

\subsection{DNS Dataset Properties}

The dataset used to evaluate the performance of various models and the development of the new models is taken from turbulent channel flow simulations of [10]. The DNS code solves the Navier-Stokes equations in spectral space with the variables discretised using Fourier decomposition in $\mathrm{x}$ and $\mathrm{z}$ while using a collocated Chebyshev discretization along $\mathrm{y}$. The domain is a rectangular box with the $\mathrm{x}, \mathrm{y}, \mathrm{z}$ extents as $L_{x}=\pi, L_{y}=2$ and $L_{z}=\pi / 2$ and the corresponding number of grid points as $N_{x}=384 ; N_{y}=385$ and $N_{z}=384$ respectively. The Reynolds number based on the friction velocity and channel half width (h) is $\operatorname{Re} \tau=u_{\tau} h / v=950$, where $u_{\tau}=\sqrt{\tau w a l l / \rho}$ is the friction velocity, $\rho$ is the fluid density and $\tau_{\text {wall }}$ is the wall shear stress. Since the data used is from a low Reynold's number DNS of turbulent channel flow, validity of the results presented should be checked at higher Reynolds numbers.

The mean of various turbulent quantities including the derivatives of velocity components, is computed by averaging along the homogeneous streamwise $\mathrm{x}$ and spanwise $\mathrm{z}$ directions and over 7 time snapshots, leading to the sample size for calculation of the statistics to be around 1 million for each point along the wall normal direction.

The calculation of 'true dissipation'(Eq.(3)) requires that derivatives of the velocity fields should be calculated using spectral methods to achieve best possible accuracy for the current DNS dataset. Derivatives of all the components of velocity are calculated in Fourier space along streamwise $\mathrm{x}$ and spanwise $\mathrm{z}$ directions, while along wall normal y direction, they are calculated by taking derivatives of Chebyshev polynomial fits of the velocity components.

The components of the fluctuating velocity gradient tensor are obtained by subtracting their mean from the instantaneous values. Divergence of the velocity field which should be 0 for incompressible flows, is used as an indicator of accuracy of the derivatives. The statistics of divergence of the instantaneous velocity field reveal the divergence to have a mean of $-2.71 \times 10^{-12}$, median $=5.586 \times 10^{-5}$ and standard deviation $=8.12 \times 10-3$, indicating sufficient accuracy of the current method to calculate the derivatives. These derivative fields will be used to evaluate the performance of various existing and new models.

\subsection{Evaluation of Model Performance}

The turbulent dissipation rate estimated for the models (em) based on local axisymmetry assumption (Eq.(5)) and weak local isotropy assumption (Eq.(7)) are plotted in Fig. 1 as a mean of the fraction of true dissipation (eT ) against the 
distance from the channel wall in viscous (+) units. While the local axisymmetry model overestimates the turbulent dissipation rate for all wall-normal locations, the weak local isotropy model performs well only in region away from the wall where $\mathrm{y}_{+}>100$ and is a poor estimate near the wall. The various terms of true turbulent dissipation rate (Eq. (3)) can be expressed as Eqs. (8)-(10) and are presented in Fig. 3.

$$
\begin{aligned}
D_{i j}=2\left\langle\frac{\left(\frac{\partial u_{i}^{\prime}}{\partial x_{j}}\right)^{2}}{\varepsilon_{T}}\right\rangle & i=j \\
C_{i j}=\left\langle\frac{\left(\frac{\partial u_{i}^{\prime}}{\partial x_{j}}\right)^{2}}{\varepsilon_{T}}\right\rangle & i \neq j \\
M_{i j}=2\left\langle\frac{\left(\frac{\partial u_{i}^{\prime}}{\partial x_{j}}\right)\left(\frac{\partial u_{j}^{\prime}}{\partial x_{i}}\right)}{\varepsilon_{T}}\right\rangle & i \neq j
\end{aligned}
$$

where $\mathrm{i}, \mathrm{j}$ indices take the values 1,2,3 denoting the $\mathrm{x}, \mathrm{y}, \mathrm{z}$ components respectively. The round brackets indicate that the notations are not as per Einstein's convention and hence no summation is implied over repeated indices.

It is clear from Fig. 3 that the turbulence quantities are not locally axisymmetric in the near wall region, indicated by the fact that all the terms of the turbulent dissipation rate are negligible near the wall $\left(\mathrm{y}^{+}<5\right)$ except for the squares of $y$ derivatives of u' and w' ( $C_{12}$ and $C_{32}$ ) and z derivative of u' $\left(C_{13}\right)$, a fact which also implies an absence of homogeneity between $\mathrm{x}-\mathrm{y} . \mathrm{y}-\mathrm{z}$ and $\mathrm{x}-\mathrm{z}$ planes (an assumption of the weak local isotropy model), hence these models perform poorly in the near wall region.

To get an estimate of the net error in the estimate of turbulent dissipation rate by the models, we define the integral ratio $\left(\xi_{m}\right)$ as the ratio of the integral of turbulent dissipation rate from the wall as calculated by the models to that of the true dissipation and is given by Eq. (11)

$$
\xi_{m}\left(y^{+}\right)=\frac{\int_{0}^{y^{+}}<\varepsilon_{m}>\left(y^{+}\right) d y^{+}}{\int_{0}^{y^{+}}<\varepsilon_{T}>\left(y^{+}\right) d y^{+}}
$$

The integral ratio $\left(\xi_{m}\right)$ for local axisymmetry model and weak local isotropy models are compared at various wallnormal locations in Fig. 2. The estimate of turbulent dissipation rate in the near wall region is poor for both the models which leads to large values of $\xi_{m}$ throughout the channel height. The integral of dissipation rate for entire channel for axisymmetric model is $\approx 1.1$ times integral of the true dissipation rate while the corresponding value is $\approx 1.3$ times for the weak isotropy model. Due to poor performance of the models in the near wall region, where the true dissipation rate is quite high, a better estimate of the turbulent dissipation rate is needed.

To arrive at an improved model, note that the weak isotropy model performs poorly in the near wall region, but is a very good estimate far away from it. The assumption of homogeneity between $x-z, y-z$ and $x-y$ planes is the major assumption in this model and the mean flow itself does not adhere to this assumption primarily in the near wall region, due to presence of the wall. Due to absence of wall in the $x-z$ plane, the homogeneity in this plane should be made independent of the wall-normal $x-y$ and $y-z$ planes. Hence, equating the $x-z$ plane cross derivative terms, we get (Eq. (12))

$$
\left\langle\left(\frac{\partial u^{\prime}}{\partial z}\right)^{2}\right\rangle=\left\langle\left(\frac{\partial v^{\prime}}{\partial z}\right)^{2}\right\rangle=\left\langle\left(\frac{\partial w^{\prime}}{\partial x}\right)^{2}\right\rangle=\left\langle\left(\frac{\partial v^{\prime}}{\partial x}\right)^{2}\right\rangle
$$

From continuity equation for incompressible flows, 


$$
\left\langle\left(\frac{\partial w^{\prime}}{\partial z}\right)^{2}\right\rangle=\left\langle\left(-\frac{\partial u^{\prime}}{\partial x}-\frac{\partial v^{\prime}}{\partial y}\right)^{2}\right\rangle
$$

Using the same assumptions as the weak isotropy formulation for the remaining terms (Eqs. (14)-(15)),

$$
\begin{gathered}
\left\langle\frac{\partial u^{\prime}}{\partial z} \frac{\partial w^{\prime}}{\partial x}\right\rangle=\left\langle\frac{\partial u^{\prime}}{\partial y} \frac{\partial v^{\prime}}{\partial x}\right\rangle=\left\langle\frac{\partial v^{\prime}}{\partial z} \frac{\partial w^{\prime}}{\partial y}\right\rangle \\
\left\langle\left(\frac{\partial w^{\prime}}{\partial y}\right)^{2}\right\rangle=\frac{1}{2}\left\langle\left(\frac{\partial u^{\prime}}{\partial y}\right)^{2}+\left(\frac{\partial v^{\prime}}{\partial x}\right)^{2}\right\rangle
\end{gathered}
$$

The estimate of turbulent dissipation rate by using new formulation $\left(\varepsilon_{N F}\right)$ is:

$$
\varepsilon_{N F}=v\left\langle 4\left(\frac{\partial u^{\prime}}{\partial x}\right)^{2}+4\left(\frac{\partial v^{\prime}}{\partial y}\right)^{2}+\frac{3}{2}\left(\frac{\partial u^{\prime}}{\partial y}\right)^{2}+\frac{9}{2}\left(\frac{\partial v^{\prime}}{\partial x}\right)^{2}+4\left(\frac{\partial u^{\prime}}{\partial x} \frac{\partial v^{\prime}}{\partial y}\right)+6\left(\frac{\partial u^{\prime}}{\partial y} \frac{\partial v^{\prime}}{\partial x}\right)\right\rangle
$$

The assumptions of this new formulation are quite valid in the near wall region as seen in Fig. 3 and performs quite well when $\mathrm{y}^{+}<5$ (Fig. 1), where the turbulent kinetic energy dissipation rate is high. A comparison of integral ratio $\left(\xi_{m}\right)$ (Fig. 2) shows that overall, the new formulation underestimates the turbulent dissipation rate while local axisymmetry or weak isotropy model overestimate it as indicated by the values of the integral ratio at channel midheight $(\approx 86 \%$ and $\approx$ $130 \%$ respectively)

An improved formulation can be arrived at by noticing from Fig. 2 that the new formulation performs really well in the near wall region $\left(\mathrm{y}^{+} \approx 3\right)$ and the weak isotropy formulation is a better estimate from $\mathrm{y}^{+} \approx 100$ upto channel mid-height. While between $\mathrm{y}^{+} \approx 3$ and $\mathrm{y}+\approx 100$, neither of them are appropriate. To combine the advantages of both the new formulation and weak isotropy assumption, the turbulent dissipation rate can be approximated by new formulation below $\mathrm{y}^{+}=3$ and by weak isotropy formulation above $\mathrm{y}+=100$ while being a linear combination of estimates from both these formulations between $\mathrm{y}^{+}=3$ and $\mathrm{y}^{+}=100$. This blend formulation is given by Eq. (17), where $\varepsilon_{B l}$ is the estimate from the blend of the new formulation $\left(\varepsilon_{N F}\right.$, Eq. (16)) and the weak isotropy assumption( $\varepsilon_{W I}$, Eq. (7)) and b is blend function given by Eq. (18)

$$
<\varepsilon_{B l}>=<\varepsilon_{N F}>(b)+<\varepsilon_{W I}>(1-b)
$$

$$
\begin{aligned}
& b\left(y^{+}\right)=1 \\
& b\left(y^{+}\right)=\left(100-y^{+}\right) / 97 \\
& b\left(y^{+}\right)=0
\end{aligned}
$$

$$
\begin{aligned}
y^{+} & <3 \\
3 \leq y^{+} & \leq 100 \\
y^{+} & <100
\end{aligned}
$$

The results from such a blend are shown in Fig. 1 and Fig. 2, demonstrating a better estimate of the true dissipation, specifically between $\mathrm{y}^{+}=3$ and $\mathrm{y}^{+}=100$. The overall dissipation in the channel as indicated by the value of $\xi_{m}$ at channel mid height is $\approx 95 \%$ of the true dissipation as compared to $\approx 86 \%$ for new formulation and $\approx 130 \%$ for theweak isotropy assumption alone. 


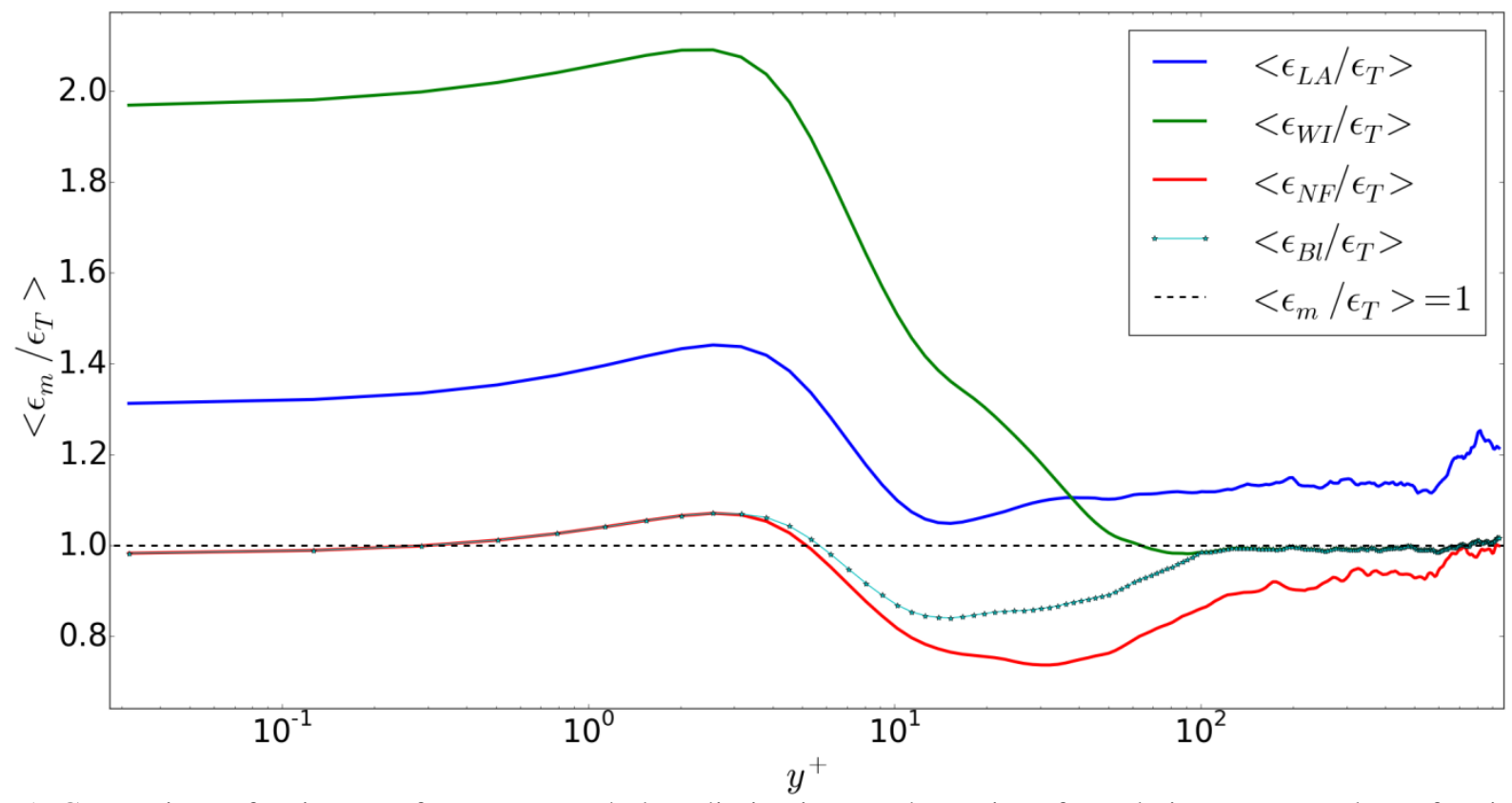

Fig. 1: Comparison of estimates of true mean turbulent dissipation rate by various formulations expressed as a fraction of it.

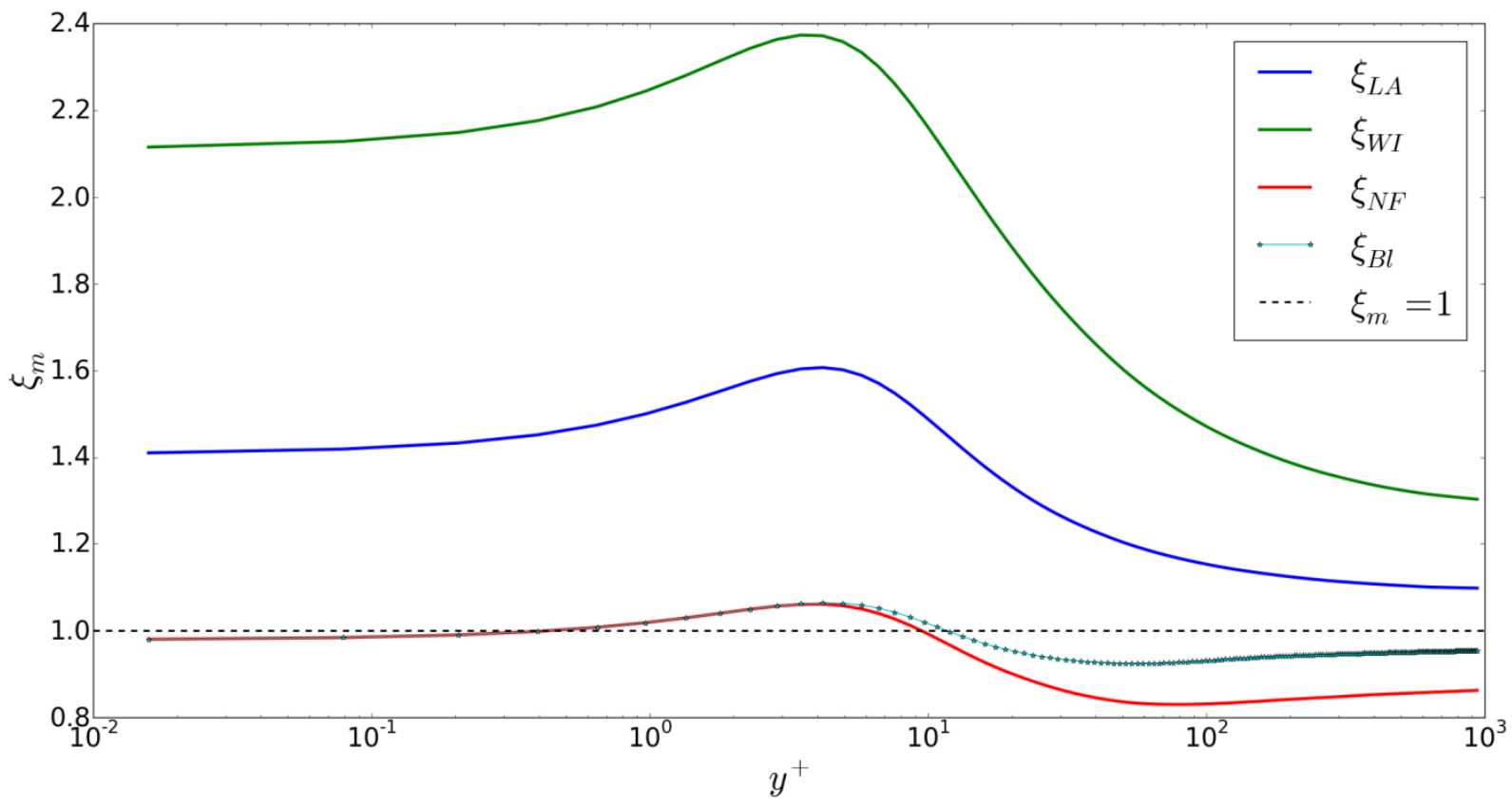

Fig. 2: Comparison of Integral Ratio (xm) values of various formulations. 


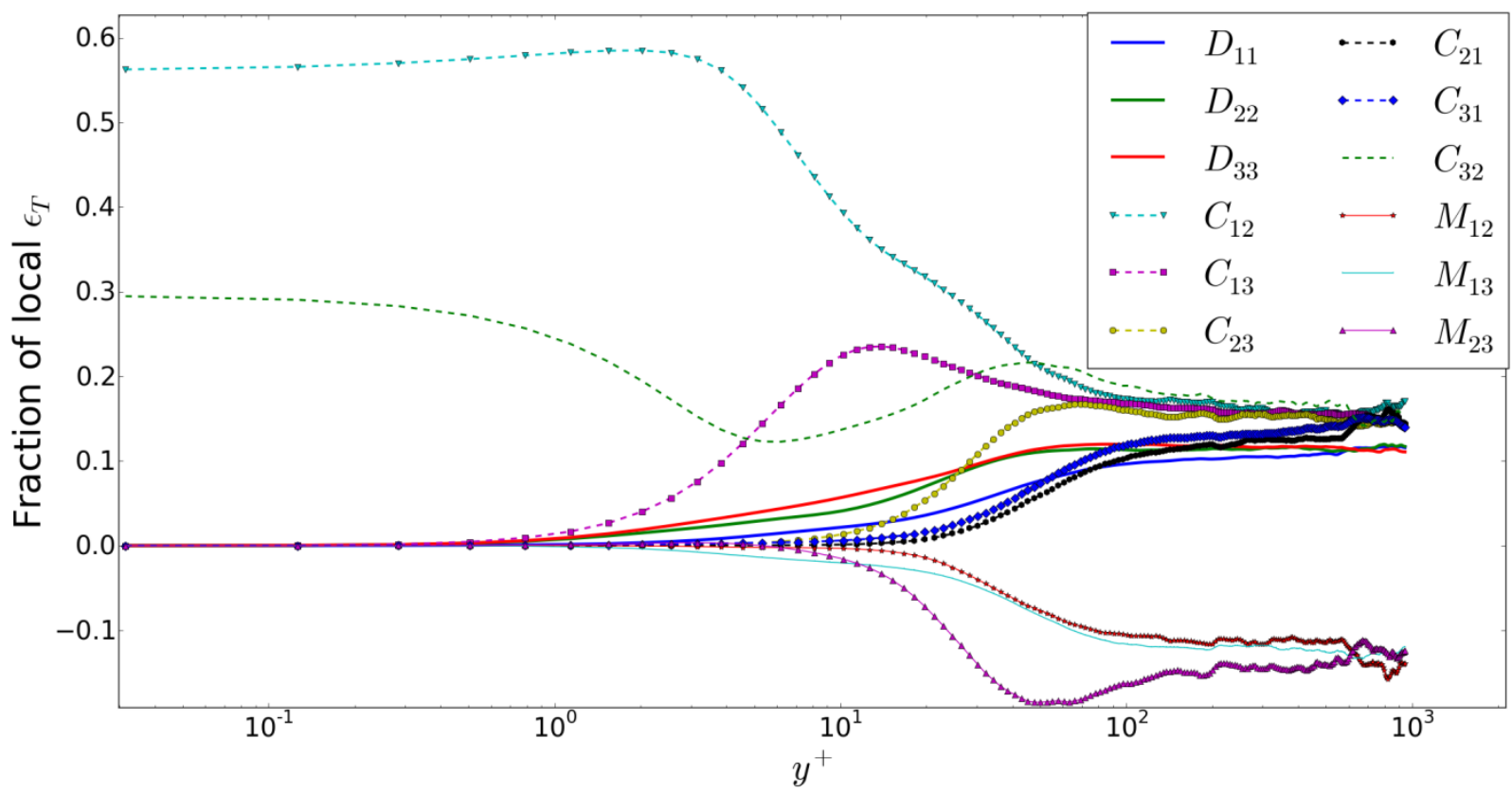

Fig. 3: Comparison of various components of local true dissipation rate, expressed a fraction of it.

\section{Conclusion}

Data from DNS simulation of a turbulent channel flow has been used to calculate and compare true turbulent dissipation rate to that as predicted by known direct estimation models used in the absence of the full velocity gradient tensor, as in 2D planar PIV measurements of turbulent flows. These models are found to perform poorly in the near wall region, overestimating the turbulent dissipation rate. A new model has been devised, that performs well in the near wall region and gives overall good dissipation estimates throughout the channel height. The maximum error in the local turbulent dissipation rate estimated by this model is $\approx 15 \%$ of true turbulent dissipation while the integral of turbulent dissipation rate for the entire channel height is $\approx 95 \%$ times the integral of true dissipation. Since the DNS dataset used for the calculations is from a low Reynolds number simulation, the performance of the models should be validated for high Reynolds number cases.

\section{References}

[1] S. B. Pope, Turbulent flows, 2001.

[2] P. Davidson, Turbulence: an introduction for scientists and engineers. Oxford University Press, 2015.

[3] W. K. George, H. J. Hussein, "Locally axisymmetric turbulence," Journal of Fluid Mechanics, vol. 233, pp. 1-23, 1991.

[4] L. Browne, R. Antonia, D. Shah, "Turbulent energy dissipation in a wake," Journal of Fluid Mechanics, vol. 179, pp. 307-326, 1987.

[5] R. Antonia, J. Kim, L. Browne, "Some characteristics of small-scale turbulence in a turbulent duct flow," Journal of Fluid Mechanics, vol. 233, pp. 369-388, 1991.

[6] K. Sharp, R. Adrian, "PIV study of small-scale flow structure around a rushton turbine," AIChE Journal, vol. 47, no. 4, pp. 766-778, 2001.

[7] A. Delafosse, M.-L. Collignon, M. Crine, D. Toye, "Estimation of the turbulent kinetic energy dissipation rate from 2d-piv measurements in a vessel stirred by an axial mixel ttp impeller," Chemical Engineering Science, vol. 66, no. 8, pp. 1728-1737, 2011.

[8] D. Xu, J. Chen, "Accurate estimate of turbulent dissipation rate using piv data," Experimental Thermal and Fluid Science, vol. 44, pp. 662-672, 2013. 
[9] P. Doron, L. Bertuccioli, J. Katz, T. Osborn, "Turbulence characteristics and dissipation estimates in the coastal ocean bottom boundary layer from piv data," Journal of Physical Oceanography, vol. 31, no. 8, pp. 2108-2134, 2001.

[10] V. Kitsios, J. Sillero, J. Frederiksen, J. Soria, "Proposed stochastic parameterisations of subgrid turbulence in large eddy simulations of turbulent channel flow," Journal of Turbulence, vol. 16, no. 8, pp. 729-741, 2015. 